\title{
ON THE STONE-ČECH BICOMPACTIFICATION OF A BISPACE
}

\author{
S. GARCIA-FERREIRA, S. ROMAGUERA and M. SANCHIS
}

(Received 6 April 1998; revised 26 February 1999)

Communicated by J. A. Hillman

\begin{abstract}
In this paper we study the Stone-Čech bicompactification $\left(\beta_{2} X, \hat{P}, \hat{Q}\right)$ of the bispace $(X, P, Q)$. We show that the ring of all continuous real-valued functions on $\left(\beta_{2} X, \hat{P} \vee \hat{Q}\right)$ may be identified with the uniform closure of a suitable subring of $C\left(\beta_{2} X, \hat{P} \vee \hat{Q}\right)$. Using this result, we give a characterization of the Wallman-Sanin compactifications of the pairwise Tychonoff bitopological spaces.

1991 Mathematics subject classification (Amer. Math. Soc.): primary 54C20, 54C30, 54D35, 54E50, 54E55.

Keywords and phrases: Bispace, Wallman-Sanin compactification, Stone-Čech bicompactification, equivalent compactifications, bicontinuous function.
\end{abstract}

\section{Introduction and preliminaries}

A bitopological space is a triple $(X, P, Q)$ where $X$ is a set and $P$ and $Q$ are topologies on $X$. A function $f:(X, P, Q) \rightarrow(Y, S, T)$ is said to be bicontinuous if both $f:(X, P) \rightarrow(Y, S)$ and $f:(X, Q) \rightarrow(Y, T)$ are continuous. The study of bitopological spaces was initiated by Kelly in [Ke]. He introduced the concepts of pairwise Hausdorffness, pairwise regularity and pairwise normality for a bitopological space. Kelly also established interesting bitopological versions of some classical topological theorems. Later, the study of bitopological spaces was continued by several topologists and classical concepts and results of the theory of spaces were extended in the context of bitopological spaces.

This paper is devoted to the study of the bicompactifications of bitopological spaces. The first notion of bitopological compactness was introduced, independently, by Fletcher, Hoyle III and Patty in [FHP] and by Kim in [Ki]: A bitopological space

The second-listed and the third-listed author were supported by DGES, grant PB95-0737.

(C) 1999 Australian Mathematical Society 0263-6115/99 \$A2.00+0.00 
$(X, P, Q)$ is called pairwise compact if every cover of $X$ consisting of $P$-open and $Q$-open subsets, with at least one non-empty $P$-open and one non-empty $Q$-open set, has a finite subcover. But, it is easy to see that this notion of bitopological compactness is not a productive property. Birsan in [Bi] and Swart [Sw] gave another bitopological compactness-like properties which allow Tychonoff product theorems. According to Birsan, a bitopological space $(X, P, Q)$ is pair compact if every $P$-open cover has a finite $Q$-open refinement, and every $Q$-open cover has a finite $P$-open refinement. By Swart's definition, a bitopological space $(X, P, Q)$ is pair compact if every cover of sets from $P \cup Q$ has a finite subcover. One can see that these two concepts differ by considering the bispace $([-1,0) \cup(0,1], u, l)$ : This space is pair compact in the sense of Birsan and is not under Swart's definition. However, in Birsan's definition, bicontinuous functions are not quasi-uniformly continuous (for details see [Sal]). Based on the fact that compactness agrees with realcompactness plus pseudocompactness, Saegrove [Sae] introduced one more bicompactness-like concept and a bicompactification of a bitopological space $(X, P, Q)$, but his bicompactification does not induce a compactification of $(X, P \vee Q)$. These historical facts and the consideration that a bitopology $(P, Q)$ essentially specifies a decomposition of the topology $P \vee Q$ into a left topology $P$ and a right topology $Q$ lead Salbany [Sa1, Sa2] to introduce a new concept of bitopological compactness: He says that a bitopological space $(X, P, Q)$ is 2-compact if ( $X, P \vee Q)$ is compact. Using Salbany's property, it is shown in [Sa1] that every pairwise Tychonoff space admits a pairwise Tychonoff bicompactification with the universal extension property of bicontinuous functions with range $(I, u, l)$, where $I$ is the unit closed interval and $u$ and $l$ are the upper and lower topologies in $\mathbb{R}$, respectively. Besides, this universal extension property holds for all bicontinuous functions $f:(X, P, Q) \rightarrow(K, S, T)$ whenever that $(K, S, T)$ is 2-compact. Actually, this bicompactification provides a Hausdorff compactification of the Tychonoff space $(X, P \vee Q)$ when $(X, P \vee Q)$ is a Hausdorff space. In this paper, we shall adopt Salbany's concept of 2-compactness plus the Hausdorff axiom on the space $(X, P \vee Q)$.

As the notions in the realm of bitopological spaces are not standard, we state the basic definitions of this paper:

A bitopological space $(X, P, Q)$ is called pairwise completely regular (this notion was introduced in [La]) if, for each $x \in X$ and for each $P$-closed set $A$ with $x \notin A$ there is a bicontinuous function $f:(X, P, Q) \rightarrow(I, u, l)$ such that $f(x)=0$ and $f(y)=1$ for every $y \in A$; and if for each $Q$-closed set $B$ with $x \notin B$ there is a bicontinuous function $g:(X, P, Q) \rightarrow(I, u, l)$ such that $g(x)=1$ and $g(y)=0$ for every $y \in B$. A bitopological space $(X, P, Q)$ is said to be pairwise Tychonoff if it is pairwise completely regular and $P \vee Q$ is a Hausdorff topology (this definition was introduced in [Sa1]). We remark that a pairwise completely regular space $(X, P, Q)$ is pairwise Tychonoff if and only if $P$ and $Q$ are $T_{0}$ topologies. Thus, if $(X, P, Q)$ is 
pairwise Tychonoff, then $(X, P \vee Q)$ is a Tychonoff space.

In the following the terms 'bispace' and 'space' will mean pairwise Tychonoff bitopological space and Tychonoff space, respectively. If $(X, P, Q)$ is a bispace and $Y \subseteq X$, then $\left(Y,\left.P\right|_{Y},\left.Q\right|_{Y}\right)$ stands for the subbispace of $(X, P, Q)$ with $\left.P\right|_{Y}=$ $\{V \cap Y: V \in P\}$ and $\left.Q\right|_{Y}=\{V \cap Y: V \in Q\}$. If $(X, P)$ is a space, then $C(X, P)$ (respectively, $C^{*}(X, P)$ ) denotes the set of all continuous (respectively, bounded continuous) functions from $(X, P)$ into $(\mathbb{R}, u \vee l)$ and if $(X, P, Q)$ is a bitopological spaces, then $B C(X, P, Q)$ (respectively, $B C^{*}(X, P, Q)$ ) will denote the set of all bicontinuous (respectively, bounded bicontinuous) functions from $(X, P, Q)$ into $(\mathbb{R}, u, l)$. A $Z_{P}$-zero set (respectively, a $Z_{Q^{-} \text {zero set) in a bitopological space }}$ $(X, P, Q)$ is a set of the form $\{x \in X: f(x) \geq 0\}$ (respectively, $\{x \in X: f(x) \leq 0\}$ ) where $f \in B C(X, P, Q)$. Notice that $Z \subseteq X$ is a $Z_{P}$-zero set (respectively, $Z_{Q^{-}}$ zero set) if $Z=f^{-1}(0)$ for some bicontinuous function $f:(X, Q, P) \rightarrow(I, u, l)$ (respectively, $f:(X, P, Q) \rightarrow(I, u, l))$. The set of all zero sets of a space $X$ is denoted by $\mathscr{Z}(X)$ and if $(X, P, Q)$ is a bispace, then $\mathscr{Z}_{P}(X)$ (or $\left.\mathscr{Z}_{P}\right)$ and $\mathscr{Z}_{Q}(X)$ (or $\mathscr{Z}_{Q}$ ) will denote the set of all $Z_{P}$-zero sets and all $Z_{Q^{-}}$-zero sets of $(X, P, Q$ ), respectively.

For a bispace $(X, P, Q)$ a 2-compact bispace $(\hat{X}, \hat{P}, \hat{Q})$ such that $X \subseteq \hat{X},\left.\hat{P}\right|_{X}=$ $P,\left.\hat{Q}\right|_{X}=Q$ and $X$ is dense in $(\hat{X}, \hat{P} \vee \hat{Q})$ will be called a bicompactification of $(X, P, Q)$. In $[\mathrm{Sal}]$ the following result was proved.

PROPOSITION 1.1. Every bispace $(X, P, Q)$ admits a unique (up to a bitopological homeomorphism fixing $X$ pointwise bicompactification $\left(\beta_{2} X, \hat{P}, \hat{Q}\right)$ satisfying the following (equivalent) properties:

(1) Every bicontinuous function $f:(X, P, Q) \rightarrow(K, S, T)$ has a bicontinuous extension to $\left(\beta_{2} X, \hat{P}, \hat{Q}\right)$, for all 2-compact bispace $(K, S, T)$.

(2) Every bicontinuous function $f:(X, P, Q) \rightarrow(I, u, l)$ has a bicontinuous extension to $\left(\beta_{2} X, \hat{P}, \hat{Q}\right)$.

(3) If $Z_{P}$ is a $P$-zero set and $Z_{Q}$ is a $Q$-zero set in $X$ disjoint from $Z_{P}$, then $c l_{\hat{P}} Z_{P} \cap c l_{\hat{Q}} Z_{Q}=\emptyset$.

The bicompactification $\left(\beta_{2} X, \hat{P}, \hat{Q}\right)$ in Proposition 1.1 is called the Stone- $\breve{C} e c h$ bicompactification of $(X, P, Q)$. We should remark that if $(X, P, Q)$ is a bispace, then $\left(\beta_{2} X, \hat{P} \vee \hat{Q}\right)$ is a compactification of the Tychonoff space $(X, P \vee Q)$.

We are mainly concerned with the study of Stone-Čech bicompactifications. The ring $C\left(\beta_{2} X, \hat{P} \vee \hat{Q}\right)$ will be identified with a more suitable set, and we characterize the compactifications of the type $\left(\beta_{2} X, \hat{P} \vee \hat{Q}\right)$ which are Wallman-Sanin compactifications. We also characterize the compactifications $\left(\beta_{2} X, \hat{P}\right)$ and $\left(\beta_{2} X, \hat{Q}\right)$ which are Wallman-Sanin compactification of the spaces $(X, P)$ and $(X, Q)$, respectively. In the case when the topologies are the same, our results reduce to the corresponding 
results of the Stone-Čech compactification of a Tychonoff space.

\section{The Stone-Čech bicompactification of a bispace}

For two bispaces $(X, P, Q)$ and $(Y, S, T)$, a function $\Phi:(X, P, Q) \rightarrow(Y, S, T)$ is said to be a bitopological homeomorphism if both $\Phi:(X, P) \rightarrow(Y, S)$ and $\Phi$ : $(X, Q) \rightarrow(Y, T)$ are homeomorphisms. Notice that if $\Phi:(X, P, Q) \rightarrow(Y, S, T)$ is a bitopological homeomorphism, then $\Phi$ is also a homeomorphism from $(X, P \vee Q)$ onto $(Y, S \vee T)$, but the converse can be fail; for instance, the identity mapping from $(\mathbb{R}, u, l)$ onto $(\mathbb{R}, l, u)$ is not a bitopological homeomorphism.

LEMMA 2.1. Let $(X, P, Q)$ be a bispace. If a space $(Y, T)$ is homeomorphic to $(X, P \vee Q)$, then there exist two topologies $T_{1}$ and $T_{2}$ in $Y$, with $T_{1} \vee T_{2}=T$, such that $(X, P, Q)$ and $\left(Y, T_{1}, T_{2}\right)$ are bitopologically homeomorphic.

PROOF. Let $\Phi:(Y, T) \rightarrow(X, P \vee Q)$ be a homeomorphism. Then, we define $T_{1}$ and $T_{2}$ by

$$
T_{1}=\left\{\Phi^{-1}(H): H \in P\right\} \quad \text { and } \quad T_{2}=\left\{\Phi^{-1}(H): H \in Q\right\} .
$$

We claim that $T=T_{1} \vee T_{2}$. Indeed, it is evident that $T_{1} \vee T_{2} \subseteq T$. Now, let $B \in T$ and fix $x \in B$. Since $\Phi$ is a homeomorphism, $\Phi(B)$ is open in $(X, P \vee Q)$. So, there exist $W_{1} \in P$ and $W_{2} \in Q$ such that $\Phi(x) \in W_{1} \cap W_{2} \subseteq \Phi(B)$. Hence, $x \in \Phi^{-1}\left(W_{1}\right) \cap \Phi^{-1}\left(W_{2}\right) \subseteq B$. This shows our claim. It is clear that $\Phi:\left(Y, T_{1}, T_{2}\right) \rightarrow$ $(X, P, Q)$ is a bitopological homeomorphism.

Let $K_{1}, K_{2}$ be two compactifications of a space $X$. We say that $K_{1} \leq K_{2}$ if there exists a continuous function $f: K_{2} \rightarrow K_{1}$ such that its restriction to $X$ is the identity map. Two compactifications of $K_{1}$ and $K_{2}$ of a space $X$ are said to be equivalent if $K_{1} \leq K_{2}$ and $K_{2} \leq K_{1}$. It is not hard to see that two compactifications $K_{1}$ and $K_{2}$ of $X$ are equivalent if and only if there is a homeomorphism between $K_{1}$ and $K_{2}$ fixing $X$ pointwise. The following result is a direct consequence of Lemma 2.1 .

COROLlaRY 2.1. Let $\left(X^{\prime}, P^{\prime}, Q^{\prime}\right)$ be a bicompactification of the bispace $(X, P, Q)$. If $(K, T)$ is a compactification of $(X, P \vee Q)$ equivalent to $\left(X^{\prime}, P^{\prime} \vee Q^{\prime}\right)$, then there exist two topologies $T_{1}$ and $T_{2}$ in $K$ such that $T=T_{1} \vee T_{2}$ and a bitopological homeomorphism from $\left(X^{\prime}, P^{\prime}, Q^{\prime}\right)$ onto $\left(K, T_{1}, T_{2}\right)$ fixing $X$ pointwise.

To give a suitable identification of the Stone-Čech bicompactification of a bispace we need the following concept and two results. 
DEFINITION 2.1. Let $X$ be a space. We say that $\mathscr{A} \subseteq C^{*}(X)$ generates a compactification $e(X, \mathscr{A})$ of $X$ if the evaluation map $e: X \rightarrow I^{\infty}$ is an embedding and $e(X, \mathscr{A})$ is the closure of the image of $X$ in $I^{\mathscr{A}}$.

If $\mathscr{A} \subseteq C^{*}(X)$, then $\langle\mathscr{A}\rangle$ will denote the smallest subring of $C^{*}(X)$ that contains $\mathscr{A}$. For proofs of the following facts the reader is referred to [Cha] (see also [CV]).

LEMMA 2.2. Let $X$ be a space and let $\mathscr{A} \subseteq C^{*}(X)$. Then,

(1) $\mathscr{A}$ generates a compactification $e(X, \mathscr{A})$ of $X$ if and only if $\langle\mathscr{A}\rangle$ separates points from closed sets in $X$.

(2) If $K X$ is a compactification of $X$ and

$$
\mathscr{A}_{K X}=\left\{f \in C^{*}(X): f \text { has a continuous extension to } K X\right\},
$$

then $\mathscr{A}_{K X}$ is a subalgebra of $C^{*}(X)$ that separates points from closed sets in $X$ and $e\left(X, \mathscr{A}_{K X}\right) \cong K X$.

(3) If $\mathscr{A}$ separates points from closed subsets in $X$, then $e\left(X, \mathscr{A}_{e(X, \mathscr{A})}\right) \cong e(X, \mathscr{A})$ and $\mathscr{A} \subseteq\langle\mathscr{A}\rangle \subseteq \mathscr{A}_{e(X, \mathscr{A})}$.

(4) If $\mathscr{A}, \mathscr{B} \subseteq C^{*}(X)$ generate compactifications of $X$, then $e(X, \mathscr{A}) \leq e(X, \mathscr{B})$ if and only if $\mathscr{A} \subseteq \mathscr{A}_{e(X, \mathscr{B})}$.

If $f \in \mathscr{A}_{K X}$, then $\hat{f}$ denotes the extension of $f$ to $K X$, and if $\mathscr{A} \subseteq \mathscr{A}_{K X}$, then $E(\mathscr{A})=\{\hat{f}: f \in \mathscr{A}\}$. The following theorem is taken from [BY].

THEOREM 2.1. For every compactification $K X$ of $X$ and for every $\mathscr{A} \subseteq C^{*}(X)$, we have that $\mathscr{A}$ generates a compactification $e(X, \mathscr{A})$ of $X$ satisfying that $e(X, \mathscr{A}) \cong$ $K X$ if and only if $\mathscr{A} \subseteq \mathscr{A}_{K X}$ and $E(\mathscr{A})$ separates points of $K X$.

We turn now to the main theorem.

THEOREM 2.2. If $(X, P, Q)$ is a bispace, then $B C^{*}(X, P, Q) \subseteq \mathscr{A}_{\left(\beta_{2} X, \hat{P} \vee \hat{Q}\right)}$ and $E\left(B C^{*}(X, P, Q)\right)$ separates points of $\left(\beta_{2} X, \hat{P} \vee \hat{Q}\right)$. Hence,

$$
e\left((X, P \vee Q), B C^{*}(X, P, Q)\right) \cong\left(\beta_{2} X, \hat{P} \vee \hat{Q}\right)
$$

Proof. Let $(X, P, Q)$ be a bispace. It follows from Proposition 1.1 that $B C^{*}(X, P$, $Q) \cup B C^{*}(X, Q, P) \subseteq \mathscr{A}_{\left(\beta_{2} X, \hat{P} \vee \hat{Q}\right)}$. To prove that $E\left(B C^{*}(X, P, Q)\right)$ separates points of $\left(\beta_{2} X, \hat{P} \vee \hat{Q}\right)$ we fix two different points $x, y \in \beta_{2} X$. We may suppose, without loss of generality, that $x \notin c l_{\hat{p}}\{y\}$. By pairwise complete regularity, there exists a bicontinuous function $f:\left(\beta_{2} X, \hat{P}, \hat{Q}\right) \rightarrow(I, u, l)$ such that $f(x)=0$ and $f(y)=1$. But, the restriction of $f$ to $X$ belongs to $B C^{*}(X, P, Q)$. By Theorem 2.1, we obtain that

$$
e\left((X, P \vee Q), B C^{*}(X, P, Q)\right) \cong\left(\beta_{2} X, \hat{P} \vee \hat{Q}\right)
$$


Observe from Theorem 2.2 that if $(X, P, Q)$ is a bispace, then

$$
\begin{aligned}
e\left((X, P \vee Q), B C^{*}(X, P, Q)\right) & \cong e\left((X, P \vee Q), B C^{*}(X, Q, P)\right) \\
& \cong\left(\beta_{2} X, \hat{P} \vee \hat{Q}\right) .
\end{aligned}
$$

For a bispace $(X, P, Q)$, we let $\mathscr{S}(X, P, Q)$ denote the set of all real-valued functions $f$ on $X$ such that $f=f_{1} \vee f_{2}$, where $f_{1} \in B C^{*}(X, P, Q)$ and $f_{2} \in$ $B C^{*}(X, Q, P)$. If no confusion is allowed, then we write $\mathscr{S}$ instead of $\mathscr{S}(X, P, Q)$. It should be remarked that $B C^{*}(X, P, Q) \cup B C^{*}(X, Q, P) \subseteq \mathscr{S}$ for every bispace $(X, P, Q)$; hence, $\mathscr{S}$ contains all real constant functions.

LEMMA 2.3. For a bispace $(X, P, Q)$, we have that $\mathscr{S}$ separates points from closed subsets in $(X, P \vee Q)$ and $\mathscr{S} \subseteq \mathscr{A}_{\left(\beta_{2} X, \hat{P} \vee \hat{Q}\right)}$. Hence, every function in $\mathscr{S}$ admits a continuous extension to $\left(\beta_{2} X, \hat{P} \vee \hat{Q}\right)$ and $e((X, P \vee Q), \mathscr{S}) \cong\left(\beta_{2} X, \hat{P} \vee \hat{Q}\right)$.

ProOF. To prove the first condition, we let $x \notin C$ where $C$ is a $(P \vee Q)$-closed set. Then, there are $V \in P$ and $U \in Q$ such that $x \in V \cap U \subseteq X \backslash C$. By pairwise complete regularity, there are $f_{1}, f_{2} \in B C^{*}(X, P, Q)$ such that $f_{1}(x)=0$, $f_{1}(y)=1$ for every $y \in X \backslash V, f_{2}(x)=1$, and $f_{2}(y)=0$ for every $y \in X \backslash U$. If $f=f_{1} \vee\left(1-f_{2}\right)$, then $f \in \mathscr{S}$ and $f(x)=0$ and $f(y)=1$ for every $y \in C$. Thus, $\mathscr{S}$ separates points from closed subsets in $(X, P \vee Q)$. We have pointed out above that $B C^{*}(X, P, Q) \cup B C^{*}(X, Q, P) \subseteq \mathscr{A}_{\left(\beta_{2} X, \hat{P} \vee \hat{Q}\right)}$. Since $f_{1} \vee f_{2}=\left(\left|f_{1}-f_{2}\right|+f_{1}+f_{2}\right) / 2$ for every $f_{1} \in B C^{*}(X, P, Q)$ and for every $f_{2} \in B C^{*}(X, Q, P)$, and $\mathscr{A}_{\left(\beta_{2} X, \hat{P} \vee \hat{Q}\right)}$ is an algebra containing the absolute value functions of its elements, we obtain that $\mathscr{S} \subseteq \mathscr{A}_{\left(\beta_{2} X, \hat{P} \vee \hat{Q}\right)}$. We have just proved that

$$
B C^{*}(X, P, Q) \subseteq \mathscr{S} \subseteq \mathscr{A}_{\left(\beta_{2} X, \hat{P} \vee \hat{Q}\right)}
$$

From Theorem $2.2, B C^{*}(X, P, Q)$ and $\mathscr{A}_{\left(\beta_{2} X, \hat{P} \vee \hat{Q}\right)}$ generate the compactification $\left(\beta_{2} X, \hat{P} \vee \hat{Q}\right)$. So, by Lemma 2.2 ,

$$
e((X, P \vee Q), \mathscr{S}) \cong\left(\beta_{2} X, \hat{P} \vee \hat{Q}\right)
$$

Next, we shall consider $C^{*}(X)$ endowed with the uniform convergence topology. In this context, we have the following results taken from [Bl].

Proposition 2.1 ([Bl, Prop. 1]). Let $\mathscr{A} \subseteq C^{*}(X)$ separate points from closed sets in $X$. Then, $f \in \mathscr{A}_{e(X, \infty)}$ if and only if $f$ belongs to the uniform closure of the subring of $C^{*}(X)$ generated by $\mathscr{A}$ and the set of all real constant functions. 
From Proposition 2.1 and the previous results we can obtain:

THEOREM 2.3. Let $(X, P, Q)$ be a bispace. Then $\mathscr{A}_{\left(\beta_{2} X, \hat{P} \vee \hat{Q}\right)}$ is the uniform closure of $\langle\mathscr{S}\rangle$ in $C^{*}(X, P \vee Q)$. That is, a function $f \in C^{*}(X, P \vee Q)$ admits a continuous extension to $C\left(\beta_{2} X, \hat{P} \vee \hat{Q}\right)$ if and only if $f$ belongs to the uniform closure of $\langle\mathscr{S}\rangle$ in $C^{*}(X, P \vee Q)$.

The Stone-Čech compactification $\beta X$ of a space $X$ is the only (up to a homeomorphism fixing $X$ pointwise) compactification $K$ of $X$ in which every bounded continuous real-valued function on $X$ admits a continuous extension to $K$ [GJ, Theorem 6.5$]$. In the category of bispaces, the results proved so far imply the following.

THEOREM 2.4. For a bispace $(X, P, Q)$ the following are equivalent.

(1) $\beta(X, P \vee Q)=\left(\beta_{2} X, \hat{P} \vee \hat{Q}\right)$;

(2) $C^{*}(X, P \vee Q)$ is the uniform closure of the smallest subring of $C^{*}(X, P \vee Q)$ that contains $\mathscr{S}$;

(3) $E(\mathscr{S})$ separates points of $\beta(X, P \vee Q)$;

(4) $E\left(B C^{*}(X, P, Q)\right)$ separates points of $\beta(X, P \vee Q)$.

From the next result we can see that any one of the conditions of Theorem 2.4 does not imply, in general, that $C^{*}(X, P \vee Q)=B C^{*}(X, P, Q)$.

THEOREM 2.5. $C^{*}(X, P \vee Q)=B C^{*}(X, P, Q)$ if and only if $P=Q$.

Proof. Suppose that $C^{*}(X, P \vee Q)=B C^{*}(X, P, Q)$. Let $V \in P$ and $W \in Q$ such that $V \cap W \neq \emptyset$. Fix $x \in V \cap W$. Since $(X, P \vee Q)$ is a Tychonoff space there is a continuous functions $f:(X, P \vee Q) \rightarrow(I, u \vee l)$ such that $f(x)=0$ and $f(y)=1$ for every $y \in X \backslash(V \cap W)$. By assumption, $f \in B C^{*}(X, P, Q)$ and so $f^{-1}([0,1)) \in P$ and $x \in f^{-1}([0,1)) \subseteq V \cap W$. Therefore, $P \vee Q=P$. Similarly we show that $P \vee Q=Q$. The converse is straightforward.

EXAMPLE 2.1. Consider a 2-compact bispace $(K, P, Q)$ with $P \neq Q$. By Theorem 2.5, $C(K, P \vee Q) \neq B C(K, P, Q)$. However, we have that $(K, P, Q)$ is 2-compact and $\beta(K, P \vee Q)=(K, P \vee Q)=\left(\beta_{2} K, \hat{P} \vee \hat{Q}\right)$.

LEMMA 2.4. Let $\emptyset \neq S \subseteq \mathbb{R}$. Then $f \in B C(S, u, l)$ (respectively $f \in B C(S, l, u)$ ) if and only if $f:(S, u \vee l) \rightarrow(\mathbb{R}, u \vee l)$ is continuous and increasing (respectively, decreasing).

PRoOF. It is clear that if $f:(S, u \vee l) \rightarrow(\mathbb{R}, u \vee l)$ is increasing, then $f \in$ $B C(S, u, l)$. Fix $f \in B C(S, u, l)$ and suppose that $f(s)<f(t)$ for $s, t \in S$ with $t<s$. Choose $a \in \mathbb{R}$ so that $f(s)<a<f(t)$. Then, $s \in f^{-1}((-\infty, a)) \in u$ and so 
$t \in f^{-1}((-\infty, a))$ which is a contradiction. The other equivalence can be proved by an analogous argument.

EXAMPLE 2.2. Consider the bispace $(\mathbb{N}, u, l)$ where $\mathbb{N}$ denotes the positive integers. We shall prove that $\left(\beta_{2} \mathbb{N}, \hat{u} \vee \hat{l}\right)$ is the one-point compactification $A(\mathbb{N})=\mathbb{N} \cup\{\star\}$ of $\mathbb{N}$ with the discrete topology. Thus, $\left(\beta_{2} \mathbb{N}, \hat{u} \vee \hat{l}\right) \neq \beta(\mathbb{N}, u \vee l)$. By Lemma 2.4, we have that every bicontinuous function $f:(\mathbb{N}, u, l) \rightarrow(I, u, l)$ is an increasing sequence in $I$. Hence, every function in $B C^{*}\left(\beta_{2} \mathbb{N}, \hat{u}, \hat{l}\right)$ can be identified with an increasing convergent sequence of $I$. Assume that $p, q \in \beta_{2} \mathbb{N}$ are distinct. Then, by Theorem 2.2, there is $f \in B C^{*}\left(\beta_{2} \mathbb{N}, \hat{u}, \hat{l}\right)$ such that $f(p) \neq f(q)$. But, $(f(n))_{n \in \mathbb{N}}$ is a convergent sequence in $I$, which is impossible. Thus, $\beta_{2} \mathbb{N} \backslash \mathbb{N}$ consists of only one point. Therefore, $\beta_{2} \mathbb{N}=A(\mathbb{N})$.

Example 2.2 also shows that it is possible to get the equality $\left(\beta_{2} X, \hat{P} \vee \hat{Q}\right)=$ $\left(\beta_{2} Y, \hat{S} \vee \hat{T}\right)$ for two different bispaces $(X, P, Q)$ and $(Y, S, T)$; in fact, the bispaces $(\mathbb{N}, u, l)$ and $(\mathbb{N}, l, u)$ satisfy the equality. So, in the bitopological context, $\left(\beta_{2} X, \hat{P} \vee\right.$ $\hat{Q})$ does not characterize the Stone-Čech bicompactification of a bispace $(X, P, Q)$. It is necessary to split $\hat{P} \vee \hat{Q}$ in a more suitable way. However, our results permit us to make an identification of $\left(\beta_{2} X, \hat{P}, \hat{Q}\right)$ from $\left(\beta_{2} X, \hat{P} \vee \hat{Q}\right)$. According to Proposition 2.2.2 of [Sa1], $\left(\beta_{2} X, \hat{P}, \hat{Q}\right)$ is initial, bitopologically, with respect to $B C^{*}\left(\beta_{2} X, \hat{P}, \hat{Q}\right)$. But, by Theorem 2.3, the elements of $B C^{*}\left(\beta_{2} X, \hat{P}, \hat{Q}\right)$ are the continuous extensions to $\left(\beta_{2} X, \hat{P} \vee \hat{Q}\right)$ of the functions of $B C^{*}(X, P, Q)$. Therefore, $\left(\beta_{2} X, \hat{P} \vee \hat{Q}\right)$ and $B C^{*}(X, P, Q)$ determine $\left(\beta_{2} X, \hat{P}, \hat{Q}\right)$.

EXAMPLE 2.3. We shall show that $\left(\beta_{2} \mathbb{R}, \hat{u} \vee \hat{l}\right) \cong(I, u \vee l)$. We identify $\mathbb{R}$ with $(0,1)$. By Lemma 2.4, a function $\left.f \in B C^{*}((0,1)), u, l\right)$ if and only if $f$ is increasing. Hence, it is clear that every function in $B C^{*}((0,1), u, l)$ has a continuous extension to $(I, u, l)$ and these extensions separate points in $(I, u, l$,$) (the identity$ map does the job for all points). In virtue of Theorem 2.1, we have that $e(((0,1), u \vee$ $\left.l), B C^{*}((0,1), u, l)\right) \cong(I, u \vee l)$. Therefore, $e\left((\mathbb{R}, u \vee l), B C^{*}(\mathbb{R}, u, l)\right) \cong(I, u \vee l)$.

It follows from Lemma 2.4 that

$$
\mathscr{S}(\mathbb{R}, u, l)=\left\{f_{1} \vee f_{2}: f_{1} \text { is increasing and } f_{2} \text { is decreasing }\right\} .
$$

By the previous example and Theorem 2.3, $C^{*}(\mathbb{R}, u \vee l)$ does not coincide with the uniform closure of $\langle\mathscr{S}(\mathbb{R}, u, l)\rangle$ in $C^{*}(\mathbb{R}, u \vee l)$. On the other hand, $C^{*}(I, u \vee l)$ is the uniform closure of $\langle\mathscr{S}(I, u, l)\rangle$ in $C^{*}(I, u \vee l)$.

EXAMPLE 2.4. Let $\kappa$ be an infinite cardinal. Let $[0, \kappa)$ denote the set of all ordinals smaller than $\kappa$. We define $\tau_{u}=\{[0, \theta): \theta<\kappa\}$ and $\tau_{l}=\{(\theta, \kappa): \theta<\kappa\}$. Then, $\left([0, \kappa), \tau_{\mu}, \tau_{l}\right)$ is a (pairwise Tychonoff) bispace and $f \in B C\left([0, \kappa), \tau_{\mu}, \tau_{l}\right)$ if and only 
if $\left.f:[0, \kappa), \tau_{u} \vee \tau_{l}\right) \rightarrow(\mathbb{R}, u \vee l)$ is continuous and increasing. Using an argument similar to the one of Example 2.3, we have that $\left(\beta_{2}[0, \kappa), \hat{\tau}_{u} \vee \hat{\tau}_{l}\right) \cong\left([0, \kappa+1], \tau_{u} \vee \tau_{l}\right)$, and if $c f(\kappa)>\omega$, then

$$
\left(\beta_{2}[0, \kappa), \hat{\tau}_{u} \vee \hat{\tau}_{l}\right) \cong \beta\left([0, \kappa), \tau_{u} \vee \tau_{l}\right) \cong\left([0, \kappa], \tau_{u} \vee \tau_{l}\right)
$$

\section{Wallman-Sanin compactifications.}

A family $\mathscr{C}$ of subsets of $X$ containing $\emptyset$ and $X$ is called a ring if it is closed under finite unions and finite intersections. If $(X, P, Q)$ is a bispace, then $\mathscr{Z}(X, P \vee Q)$, $\mathscr{Z}_{P}$ and $\mathscr{Z}_{Q}$ are examples of rings. If $\mathscr{C}$ is a family of subsets of $X$, then $r(\mathscr{C})$ will denote the smallest ring that contains $\mathscr{C}$. Let $X$ be a space and let $\mathscr{C}$ be a closed base for $X$ that is a ring. The space

$$
w(X, \mathscr{C})=\{\mathscr{F}: \mathscr{F} \text { is a } \mathscr{C} \text {-ultrafilter }\}
$$

with the closed base $\{\tilde{C}: C \in \mathscr{C}\}$, where $\widetilde{C}=\{\mathscr{F} \in w(X, \mathscr{C}): C \in \mathscr{F}\}$ for every $C \in \mathscr{C}$, is a $T_{1}$ compact space called the Wallman-Sanin compactification of $X$ with respect to $\mathscr{C}$ (see [Cha, Na]). In general, $w(X, \mathscr{C}$ ) is not a compactification of $X$, it is necessary to add some conditions to the closed base ring $\mathscr{C}$. Indeed, if $\mathscr{C}$ is a closed base ring that satisfies:

(1) ( $T_{1}$-base) if $F$ is closed and $x \notin F$, then there is $C \in \mathscr{C}$ such that $x \in C$ and $C \cap F=\emptyset$,

then we may define a continuous function $\psi: X \rightarrow w(X, \mathscr{C})$ by $\psi(x)=\{C \in \mathscr{C}:$ $x \in C\}$, and if $X$ is a $T_{1}$-space, then $\psi$ is an embedding. As it was showed by Sanin (Sanin's Theorem) the Wallman-Sanin compactification of $X$ with respect to $\mathscr{C}$ is the only one satisfying the following conditions:

(WS1) The family $\left\{c l_{w(X, \mathscr{C})} C: C \in \mathscr{C}\right\}$ is a base for closed sets in $w(X, \mathscr{C})$.

(WS2) If $C_{1}, \ldots, C_{n} \in \mathscr{C}$, then

$$
c l_{w(X, \mathscr{C})} \bigcap\left\{C_{i}: 1 \leq i \leq n\right\}=\bigcap\left\{c l_{w(X, \mathscr{C})} C_{i}: 1 \leq i \leq n\right\} .
$$

(WS3) If $p \in w(X, \mathscr{C}) \backslash X,\{p\}$ is closed.

(WS4) For every $C \in \mathscr{C}, c l_{w(x, \mathscr{C})} C=\widetilde{C}$.

A closed $T_{1}$-base ring $\mathscr{C}$ of $X$ is called a normal base of $X$

(2) if $A_{1}, A_{2} \in \mathscr{C}$ and $A_{1} \cap A_{2}=\emptyset$, then there are $C_{1}, C_{2} \in \mathscr{C}$ such that $A_{1} \cap C_{1}=$ $A_{2} \cap C_{2}=\emptyset$ and $X=C_{1} \cup C_{2}$.

Observe that if $X$ is a $T_{1}$-space and has a normal base $\mathscr{C}$, then $X$ is a Hausdorff space and $w(X, \mathscr{C})$ is a Hausdorff compactification of $X$. Conversely, if $X$ is a $T_{1}$-space and 
$w(X, \mathscr{C})$ is a Hausdorff compactification of $X$, then $\mathscr{C}$ is a normal base of $X$. For the details of the proofs of the facts of this paragraph the reader is referred to [Cha, $\mathrm{Na}$ ].

If $X$ is a space and $\mathscr{B} \subseteq C(X)$, then we put

$$
\mathscr{Z}(\mathscr{B})=\left\{Z \in \mathscr{Z}(X): Z=f^{-1}(0) \text { for some } f \in \mathscr{B}\right\} .
$$

LEMMA 3.1. For every bispace $(X, P, Q)$ we have that

$$
\mathscr{Z}\left(\left\langle B C^{*}(X, P, Q)\right\rangle\right)=r\left(\mathscr{Z}_{P} \cup \mathscr{Z}_{Q}\right),
$$

where (\rangle is taken inside of $C^{*}(X, P \vee Q)$ and $r()$ is taken in $\mathscr{Z}(X, P \vee Q)$.

PRoOF. Let $Z \in \mathscr{Z}\left(\left\langle B C^{*}(X, P, Q)\right\rangle\right)$ and let $f \in\left\langle B C^{*}(X, P, Q)\right\rangle$ such that $Z=f^{-1}(0)$. Then, $f=\sum_{j \leq n} f_{0}^{j} \cdot \ldots \cdot f_{k_{j}}^{j}$, where $f_{i}^{j}:(X, P, Q) \rightarrow(I, u, l)$ is bicontinuous for every $i \leq k_{j}$ and for every $j \leq n$. Then, we have that

$$
Z=\bigcap_{j \leq n}\left(\bigcup_{i \leq k_{j}}\left(\left\{x \in X: f_{i}^{j}(x) \geq 0\right\} \cap\left\{x \in X: f_{i}^{j}(x) \leq 0\right\}\right)\right),
$$

and hence $Z \in r\left(\mathscr{Z}_{P} \cup \mathscr{Z}_{Q}\right)$. Thus, $\mathscr{Z}\left(\left\langle B C^{*}(X, P, Q)\right\rangle\right) \subseteq r\left(\mathscr{Z}_{P} \cup \mathscr{Z}_{Q}\right)$. The proof of $\mathscr{Z}\left(\left\langle B C^{*}(X, P, Q)\right\rangle\right) \supseteq r\left(\mathscr{Z}_{P} \cup \mathscr{Z}_{Q}\right)$ is left to the reader.

We omit the proof of the following lemma.

LemMa 3.2. Let $(X, P, Q)$ be a bispace. If $Z \in \mathscr{Z}\left(\left\langle E\left(B C^{*}(X, P, Q)\right)\right\rangle\right)$, then $Z \cap X \in \mathscr{Z}\left(\left\langle B C^{*}(X, P, Q)\right\rangle\right)$.

We shall need in the sequel the following results:

PROPOSITION 3.1 (Taĭmanov, [Ta]). Let $Y$ be a dense subspace of a topological space $X$ and let $\psi$ be a continuous function from $Y$ into a compact Hausdorff space $Z$. Suppose that $\mathscr{M}$ is a base for the closed sets of $Z$ which is closed under finite intersections. Then $\psi$ can be continuously extended over $X$ if and only if for every pair $M_{1}, M_{2}$ of disjoint elements of $\mathscr{M}$ the inverse images $\psi^{-1}\left(M_{1}\right)$ and $\psi^{-1}\left(M_{2}\right)$ have disjoint closures in $X$.

LEMMA 3.3. For every bispace $(X, P, Q), \mathscr{Z}\left(\left\langle E\left(B C^{*}(X, P, Q)\right)\right\rangle\right)$ is a closed base for $\left(\beta_{2} X, \hat{P} \vee \hat{Q}\right)$.

PROOF. By Theorem $2.2,\left\langle E\left(B C^{*}(X, P, Q)\right)\right\rangle$ separates points in $\left(\beta_{2} X, \hat{P} \vee \hat{Q}\right)$ and this implies that $\left\langle E\left(B C^{*}(X, P, Q)\right)\right\rangle$ separates points from closed sets in $\left(\beta_{2} X, \hat{P} \vee \hat{Q}\right)$ (by Lemma 2.1 of $[C V])$. Hence, $\mathscr{Z}\left(\left\langle E\left(B C^{*}(X, P, Q)\right)\right\rangle\right)$ is a closed base for $\left(\beta_{2} X, \hat{P} \vee \hat{Q}\right)$. 
THEOREM 3.1. For every bispace $(X, P, Q)$, we have that

$$
\left(\beta_{2} X, \hat{P} \vee \hat{Q}\right) \leq w\left((X, P \vee Q), r\left(\mathscr{Z}_{P} \cup \mathscr{Z}_{Q}\right)\right)
$$

PROOF. We shall show that the inclusion map

$$
\psi: X \rightarrow\left(\beta_{2} X, \hat{P} \vee \hat{Q}\right)
$$

extends continuously to $w\left((X, P \vee Q), r\left(\mathscr{Z}_{P} \cup \mathscr{Z}_{Q}\right)\right)$. By Lemma 3.3, we have that $\mathscr{Z}\left(\left\langle E\left(B C^{*}(X, P, Q)\right)\right\rangle\right)$ is a closed base for $\left(\beta_{2} X, \hat{P} \vee \hat{Q}\right)$. Let $A, B \in$ $\mathscr{Z}\left(\left\langle E\left(B C^{*}(X, P, Q)\right)\right\rangle\right)$ with $A \cap B=\emptyset$. In virtue of Lemma $3.2, \psi^{-1}(A)=$ $X \cap A \in \mathscr{Z}\left(\left\langle B C^{*}(X, P, Q)\right\rangle\right)$ and $\psi^{-1}(B) \in \mathscr{Z}\left(\left\langle B C^{*}(X, P, Q)\right\rangle\right)$. Hence, by Lemma 3.1, $\psi^{-1}(A), \psi^{-1}(B) \in r\left(\mathscr{Z}_{P} \cup \mathscr{Z}_{Q}\right)$ and so, by (WS2),

$$
\emptyset=c l_{w\left((X, P \vee Q), r\left(\mathscr{X}_{P} \cup \mathscr{X}_{Q}\right)\right)} \psi^{-1}(A) \cap c l_{w\left((X, P \vee Q), r\left(\mathscr{X}_{P} \cup \mathscr{X}_{Q}\right)\right)} \psi^{-1}(B) .
$$

By Tămanov's theorem $\psi$ has a continuous extension to $w\left((X, P \vee Q), r\left(\mathscr{Z}_{P} \cup \mathscr{Z}_{Q}\right)\right)$.

QUESTION 3.1. Give an example which points out that it is possible to have the situation: $\left(\beta_{2} X, \hat{P} \vee \hat{Q}\right)<w\left((X, P \vee Q), r\left(\mathscr{Z}_{P} \cup \mathscr{Z}_{Q}\right)\right)$.

Now we will characterize when the equality holds in Theorem 3.1.

THEOREM 3.2. Let $(X, P, Q)$ be a bispace. The following assertions are equivalent:

(1) $\left(\beta_{2} X, \hat{P} \vee \hat{Q}\right)=w\left((X, P \vee Q), r\left(\mathscr{Z}_{P} \cup \mathscr{Z}_{Q}\right)\right)$;

(2) the following equality holds

$$
c l_{\left(\beta_{2} X, \hat{P} \vee \hat{Q}\right)} Z_{1} \cap c l_{\left(\beta_{2} X, \hat{P} \vee \hat{Q}\right)} Z_{2}=c l_{\left(\beta_{2} X, \hat{P} \vee \hat{Q}\right)}\left(Z_{1} \cap Z_{2}\right)
$$

whenever either $Z_{1}, Z_{2}$ belong to $\mathscr{Z}_{P}$ or $Z_{1}, Z_{2}$ belong to $\mathscr{Z}_{Q}$;

(3) $c l_{\left(\beta_{2} X, \hat{P} \vee \hat{Q}\right)} Z_{1}$ and $c l_{\left(\beta_{2} X, \hat{P} \vee \hat{Q}\right)} Z_{2}$ are pairwise disjoint whenever $Z_{1}, Z_{2}$ are pairwise disjoint zero-sets either in $\mathscr{Z}_{P}$ or in $\mathscr{Z}_{Q}$.

PROOF. We get that (1) implies (2) as an easy consequence of Sanin's Theorem and the implication (2) implies (3) is obvious. So, it suffices to prove that (3) implies (1).

(3) implies (1): By Theorem 3.1, it suffices to show that

$$
w\left((X, P \vee Q), r\left(\mathscr{Z}_{P} \cup \mathscr{Z}_{Q}\right)\right) \leq\left(\beta_{2} X, \hat{P} \vee \hat{Q}\right) .
$$

To do this, we have to prove that the inclusion map

$$
\psi: X \rightarrow w\left((X, P \vee Q), r\left(\mathscr{Z}_{P} \cup \mathscr{Z}_{Q}\right)\right)
$$

has a continuous extension to $\left(\beta_{2} X, \hat{P} \vee \hat{Q}\right)$. According to Proposition 3.1, we need to prove 
(a) $c l_{\left(\beta_{2} X, \hat{P} \vee \hat{Q}\right)} Z_{1} \cap c l_{\left(\beta_{2} X, \hat{P} \vee \hat{Q}\right)} Z_{2}=\emptyset$ for all pairwise disjoint elements $Z_{1}, Z_{2}$ belong to $r\left(\mathscr{Z}_{P} \cup \mathscr{Z}_{Q}\right)$, and

(b) $w\left((X, P \vee Q), r\left(\mathscr{Z}_{P} \cup \mathscr{Z}_{Q}\right)\right)$ is Hausdorff.

To prove (a), let $Z_{1}=\bigcup_{i=1}^{n_{1}} H_{i}, Z_{2}=\bigcup_{j=1}^{n_{2}} H_{j}$, with $H_{i}, H_{j} \in \mathscr{Z}_{P} \cup \mathscr{Z}_{Q}$ for $i=1, \ldots, n_{1}, j=1, \ldots, n_{2}$. By (3),

$$
c l_{\left(\beta_{2} X, \hat{P} \vee \hat{Q}\right)} H_{i} \cap c l_{\left(\beta_{2} X, \hat{P} \vee \hat{Q}\right)} H_{j}=\emptyset .
$$

Then (a) follows easily. Now, to prove (b), consider two pairwise disjoint elements, $Z_{1}$ and $Z_{2}$, of $r\left(\mathscr{Z}_{P} \cup \mathscr{Z}_{Q}\right)$. Because $c l_{\left(\beta_{2} X, \hat{P} \vee \hat{Q}\right)} Z_{1}, c l_{\left(\beta_{2} X, \hat{P} \vee \hat{Q}\right)} Z_{2}$ are pairwise disjoint compact sets, we can find $f \in C\left(\left(\beta_{2} X, \hat{P} \vee \hat{Q}\right)\right)$ such that $f\left(c l_{\left(\beta_{2} X, \hat{P} \vee \hat{Q}\right)} Z_{1}\right)=0$ and $f\left(c l_{\left(\beta_{2} X, \hat{P} \vee \hat{Q}\right)} Z_{2}\right)=1$. Since by Theorem $\left.3.1 f\right|_{X}$ admits a continuous extension to $w\left((X, P \vee Q), r\left(\mathscr{Z}_{P} \cup \mathscr{Z}_{Q}\right)\right)$, we have that $c l_{w\left((X, P \vee Q), r\left(\mathscr{Z}_{P} \cup \mathscr{Z}_{Q}\right)\right)} Z_{1}$ and $c l_{w\left((X, P \vee Q), r\left(\mathscr{X}_{P} \cup \mathscr{Z}_{Q))}\right.\right.} Z_{2}$ are completely separated in $w\left((X, P \vee Q), r\left(\mathscr{Z}_{P} \cup \mathscr{Z}_{Q}\right)\right)$. As the family $\left\{c l_{w\left((X, P \vee Q), r\left(\mathscr{X}_{P} \cup \mathscr{X}_{Q}\right)\right)} Z\right\}$, where $Z \in r\left(\mathscr{Z}_{P} \cup \mathscr{Z}_{Q}\right)$ is a base for closed sets in the compact space $w\left((X, P \vee Q), r\left(\mathscr{Z}_{P} \cup \mathscr{Z}_{Q}\right)\right)$, we have that the ring

$$
\begin{aligned}
\mathscr{R}= & \left\{f \in C \left(w\left((X, P \vee Q), r\left(\mathscr{Z}_{P} \cup \mathscr{Z}_{Q}\right)\right):\left.f\right|_{X}\right.\right. \text { admits a } \\
& \text { continuous extension to } \left.w\left((X, P \vee Q), r\left(\mathscr{Z}_{P} \cup \mathscr{Z}_{Q}\right)\right)\right\}
\end{aligned}
$$

separates points in $w\left((X, P \vee Q), r\left(\mathscr{Z}_{P} \cup \mathscr{Z}_{Q}\right)\right)$. Thus,

$$
w\left((X, P \vee Q), r\left(\mathscr{Z}_{P} \cup \mathscr{Z}_{Q}\right)\right)
$$

is Hausdorff and the proof is complete.

Let $(X, P, Q)$ be a bispace. From [Sal, Proposition 2.2.11], the families $\mathscr{Z}_{P}$ and $\mathscr{Z}_{Q}$ are bases for closed sets in $(X, P)$ and $(X, Q)$, respectively. Beginning from this fact it seems worthy of study when the topology of $w\left((X, P \vee Q), r\left(\mathscr{Z}_{P} \cup \mathscr{Z}_{Q}\right)\right)$ can be splitted in two topologies $\mathscr{U}$ and $\mathscr{L}$ such that the families

$$
\left\{c l_{w\left((X, P \vee Q), r\left(\mathscr{X}_{P} \cup \mathscr{X}_{Q}\right)\right)} P: P \in \mathscr{Z}_{P}\right\}, \quad\left\{c l_{w\left((X, P \vee Q), r\left(\mathscr{X}_{P} \cup \mathscr{X}_{Q}\right)\right)} Q: Q \in \mathscr{Z}_{Q}\right\}
$$

are (sub)bases for closed sets in $\left(w\left(X, r\left(\mathscr{Z}_{P} \cup \mathscr{Z}_{Q}\right)\right), \mathscr{U}\right)$ and $\left(w\left(X, r\left(\mathscr{Z}_{P} \cup \mathscr{Z}_{Q}\right)\right), \mathscr{L}\right)$, respectively (here $w\left(X, r\left(\mathscr{Z}_{P} \cup \mathscr{Z}_{Q}\right)\right)$ means for the set of $r\left(\mathscr{Z}_{P} \cup \mathscr{Z}_{Q}\right)$-ultrafilter). We close by turning our attention to this question. First, a lemma. We will denote by $\mathscr{Z}_{u}$ (respectively, $\mathscr{Z}_{l}$ ) the family of all $Z_{u}$-zero sets (respectively, $Z_{l}$-zero sets) in $(I, u, l)$.

LEMMA 3.4. A set $Z \subset I$ belongs to $\mathscr{Z}_{u}$ (respectively, to $\mathscr{Z}_{l}$ ) if and only if there exists $\alpha \in I$ such that $Z=f^{-1}([\alpha, 1])$ (respectively, $Z=f^{-1}([0, \alpha])$ for some bicontinuous function from $(I, u, l)$ into $(I, l, u)$ (respectively, into $(I, u, l)$ ). 
PROOF. We only prove the case concerning $\mathscr{Z}_{u}$; the other one follows from a similar argument. Suppose first that $Z \in \mathscr{Z}_{u}$. Then $Z=f^{-1}(0)$ for some bicontinuous function $f$ from $(I, u, l)$ into $(I, l, u)$. By Lemma $2.4, f$ is a decreasing function. So, if $\alpha=\inf \{x \in I \mid f(x)=0\}$, it is clear that $f(x)=0$ if and only if $x \geq \alpha$. Conversely, let $\alpha \in I$. We can suppose, without loss of generality that $\alpha \neq 0$. Consider the bicontinuous function $f$ from $(I, u, l)$ into $(I, l, u)$ defined by the requirement that $f(x)$ be $1-x$ whenever $x \in I$ and let $\beta=f(\alpha)$. Let $g$ be the bicontinuous function from $(I, l, u)$ into $(I, l, u)$ defined as follows:

$$
g(x)= \begin{cases}0 & \text { if } x \leq \beta \\ (x-\beta) /(1-\beta) & \text { if } x \geq \beta\end{cases}
$$

It is easy to check that $(g \circ f)^{-1}(0)=[\alpha, 1]$.

THEOREM 3.3. Let $(X, P, Q)$ be a bispace. The following assertions are equivalent:

(1) $\left(w\left(X, r\left(\mathscr{Z}_{P} \cup \mathscr{Z}_{Q}\right)\right), \mathscr{U}, \mathscr{L}\right)$ is pairwise Tychonoff.

(2) $\left(w\left(X, r\left(\mathscr{Z}_{P} \cup \mathscr{Z}_{Q}\right)\right), \mathscr{U}, \mathscr{L}\right)$ is bitopological homeomorphic to $\left(\beta_{2} X, \hat{P}, \hat{Q}\right)$.

PROOF. The implication (2) implies (1) is clear. So, we only need to prove that (1) implies (2).

(1) implies (2): First notice that, because $\left(w\left(X, r\left(\mathscr{Z}_{P} \cup \mathscr{Z}_{Q}\right)\right), \mathscr{U}, \mathscr{L}\right)$ is pairwise Tychonoff and the usual topology in $w\left((X, P \vee Q), r\left(\mathscr{Z}_{P} \cup \mathscr{Z}_{Q}\right)\right)$ is finer than $\mathscr{U} \vee \mathscr{L}$, the (Hausdorff) space $\left(w\left(X, r\left(\mathscr{Z}_{P} \cup \mathscr{Z}_{Q}\right)\right), \mathscr{U} \vee \mathscr{L}\right)$ is compact. So, by Proposition 1.1 , it suffices to show that every bicontinuous function $f:(X, P, Q) \rightarrow(I, u, l)$ admits a bicontinuous extension to $\left(w\left(X, r\left(\mathscr{Z}_{P} \cup \mathscr{Z}_{Q}\right)\right), \mathscr{U}, \mathscr{L}\right)$. Let $f$ be as above. From now on, we shall identify the points of $w\left((X, P \vee Q), r\left(\mathscr{Z}_{P} \cup \mathscr{Z}_{Q}\right)\right)$ with the $r\left(\mathscr{Z}_{P} \cup \mathscr{Z}_{Q}\right)$-ultrafilters (in $\left.X\right)$ : a point $x \in X$ is identified with the (only) $r\left(\mathscr{Z}_{P} \cup \mathscr{Z}_{Q}\right)$-ultrafilter $\mathscr{D}$ satisfying that $Z \in \mathscr{D}$ if and only if $x \in Z$, and the points of $w\left((X, P \vee Q), r\left(\mathscr{Z}_{P} \cup \mathscr{Z}_{Q}\right)\right) \backslash X$ are identified with the free $r\left(\mathscr{Z}_{P} \cup \mathscr{Z}_{Q}\right)$-ultrafilters. In this way, if $Z \in r\left(\mathscr{Z}_{P} \cup \mathscr{Z}_{Q}\right)$, we have that $p \in c l_{w\left((X, P \vee Q), r\left(\mathscr{X}_{P} \cup \mathscr{X}_{Q}\right)\right)} Z$ if and only if $Z \in p$ (WS4). Now, for each $p \in w\left((X, P \vee Q), r\left(\mathscr{Z}_{P} \cup \mathscr{Z}_{Q}\right)\right)$ define $p_{1}, p_{2}$ as follows:

$$
p_{1}=\left\{Z \in p: Z \in Z_{P}\right\}, \quad p_{2}=\left\{Z \in p: Z \in Z_{Q}\right\},
$$

and consider the families

$$
\begin{aligned}
\mathscr{H}(f, p) & =\left\{Z_{u} \in \mathscr{Z}_{u}: f^{-1}\left(Z_{u}\right) \in p_{1}\right\}, \\
\mathscr{F}(f, p) & =\left\{Z_{l} \in \mathscr{Z}_{l}: f^{-1}\left(Z_{l}\right) \in p_{2}\right\} .
\end{aligned}
$$


Let $p \in w\left((X, P \vee Q), r\left(\mathscr{Z}_{P} \cup \mathscr{Z}_{Q}\right)\right)$. Denote by $f^{*}(p)$ the subset

$$
\bigcap\left\{Z_{u} \cap Z_{l}: Z_{u} \in \mathscr{H}(f, p), Z_{l} \in \mathscr{F}(f, p)\right\} .
$$

Since $(I, u \vee l)$ is compact, $f^{*}(p)$ is nonempty. We shall prove that the subset $f^{*}(p)$ is a singleton. Suppose the contrary, we claim that there exist $x, y \in f^{*}(p)$ with $x<y$. Let $U$ and $V$ pairwise disjoint basic open sets in $u$ and in $l$, respectively, such that $x \in U$ and $y \in V$. By Lemma 3.4 we have that

$$
I \backslash U \in \mathscr{Z}_{u}, \quad I \backslash V \in \mathscr{Z}_{l} .
$$

Since $X=f^{-1}(I \backslash U) \cup f^{-1}(I \backslash V)$, applying that $p$ is a $r\left(\mathscr{Z}_{P} \cup \mathscr{Z}_{Q}\right)$-ultrafilter, $f^{-1}(I \backslash U) \in p$ or $f^{-1}(I \backslash V) \in p$. So, $f^{-1}(I \backslash U) \in p_{1}$ or $f^{-1}(I \backslash V) \in p_{2}$. Since $x, y \in f^{*}(p)$ we have that $x \in(I \backslash U) \cap U$ or $y \in(I \backslash V) \cap V$, a contradiction. Thus, $f^{*}(p)$ is a singleton.

Define now $g:\left(w\left(X, r\left(\mathscr{Z}_{P} \cup \mathscr{Z}_{Q}\right)\right), \mathscr{U}, \mathscr{L}\right) \rightarrow(I, u, l)$ as $g(p)=f^{*}(p)$. It is easy to check that $g$ is an extension of $f$ to $\left(w\left(X, r\left(\mathscr{Z}_{P} \cup \mathscr{Z}_{Q}\right)\right), \mathscr{U}, \mathscr{L}\right)$. We shall prove that $g$ is bicontinuous. We only show that $g:\left(w\left(X, r\left(\mathscr{Z}_{P} \cup \mathscr{Z}_{Q}\right)\right), \mathscr{U}\right) \rightarrow(I, u)$ is continuous, the other case follows from a similar argument. Let $p \in w((X, P \vee$ $\left.Q), r\left(\mathscr{Z}_{P} \cup \mathscr{Z}_{Q}\right)\right)$. Consider $U \in u$ such that $g(p) \in U$. By Lemma 3.4, we can find two pairwise disjoint subsets $W \in u$ and $V \in l$ such that $I \backslash W \in \mathscr{Z}_{u}, I \backslash V \in \mathscr{Z}_{l}$, $g(p) \in W$ and $I \backslash V \subset U$. Since $X=g^{-1}(I \backslash W) \cup g^{-1}(I \backslash V)$, we have that $w\left(X, r\left(\mathscr{Z}_{P} \cup \mathscr{Z}_{Q}\right)\right)=c l_{w\left((X, P \vee Q), r\left(\mathscr{X}_{p} \cup \mathscr{Z}_{Q}\right)\right)} g^{-1}(I \backslash W) \cup c l_{w\left((X, P \vee Q), r\left(\mathscr{X}_{P} \cup \mathscr{X}_{Q}\right)\right)} g^{-1}(I \backslash V)$.

Now, according to the definition of $g(p)$, if $g^{-1}(I \backslash W) \in p_{1}$, then $g(p) \in g^{-1}(I \backslash W)$ that does not make sense since $g(p) \in W$. So, $g^{-1}(I \backslash W) \notin p_{1}$ and, a posteriori, $g^{-1}(I \backslash W) \notin p$. So, $w\left(X, r\left(\mathscr{Z}_{P} \cup \mathscr{Z}_{Q}\right)\right) \backslash c l_{w\left((X, P \vee Q), r\left(\mathscr{I}_{P} \cup \mathscr{Z}_{Q}\right)\right)} g^{-1}(I \backslash W)$ is a $\mathscr{U}$-open neighborhood of $p$. Because $W \cap V=\emptyset$ and $I \backslash V \subset U$, we obtain that

$$
g\left(w\left(X, r\left(\mathscr{Z}_{P} \cup \mathscr{Z}_{Q}\right)\right) \backslash c l_{w\left((X, P \vee Q), r\left(\mathscr{X}_{P} \cup \mathscr{P}_{Q}\right)\right)} g^{-1}(I \backslash W)\right) \subset U .
$$

This completes the proof.

QUESTION 3.2. Give an example of a bispace $(X, P, Q)$ such that $\left(\beta_{2} X, \hat{P}, \hat{Q}\right)$ is different from $\left(w\left(X, r\left(\mathscr{Z}_{P} \cup \mathscr{Z}_{Q}\right)\right), \mathscr{U}, \mathscr{L}\right)$.

\section{References}

[BY] B.J. Ball and S. Yokura, 'Compactifications determined by subsets of $C^{*}(X)$, II', Topol. Appl. 15 (1983), $1-6$.

[Bi] T. Birsan, 'Compacité dans les espaces bitopologiques', An. Sti. Univ. Al. I. Cuza, Mat. 15 (1969), 317-328. 
[B1] J. L. Blasco, 'Hausdorff compactifications and Lebesgue sets', Topol. Appl. 15 (1993), 111-117.

[CV] A. Caterino and M. C. Vipera, 'Weight of a compactification and generating sets of functions', Rend. Sem. Math. Univ. Padova 79 (1988), 37-47.

[Cha] R. E. Chandler, Hausdorff compactifications, Lecture Notes in Pure and Appl. Math., vol. 23 (Marcel Dekker, 1976).

[En] R. Engelking, General topology (PWN, Warsaw, 1977).

[FHP] P. Fletcher, H. B. Hoyle III and C. W. Patty, 'The comparison of topologies', Duke Math. J. 36 (1969), 325-331.

[GJ] L. Gillman and J. Jerison, Rings of continuous functions (Van Nostrand Reinhold, New York, 1960).

[Ke] J. C. Kelley, 'Bitopological spaces', Proc. London Math. Soc. 13 (1963), 71-89.

[Ki] Y. W. Kim, 'Pairwise compactness', Publ. Math. Debrecen 15 (1968), 87-90.

[La] E. P. Lane, 'Bitopological spaces and quasi-uniform spaces', Proc. London Math. Soc. 17 (1967), 341-356.

[Na] J. Nagata, Modern general topology (North-Holland, Amsterdam, 1968).

[Sae] M. J. Saegrove, 'Pairwise complete regularity and compactification', J. London Math. Soc. 7 (1973), 286-290.

[Sa1] S. Salbany, Bitopological spaces, compactifications and completions ( $\mathrm{Ph} . \mathrm{D}$. Thesis, Univ. Cape Town, 1970).

[Sa2] - 'A bitopological view of topology and order', in: Categorical Topology (Proc. Toledo, Ohio, 1983) (Heldermann, 1984) pp. 481-504.

[St] E. F. Steiner, 'Normal families and completely regular spaces', Duke Math. J. 33 (1966), 743-746.

[Sw] J. Swart, 'Total disconnectedness in bitopological spaces and product of bitopological spaces', Proc. Kon. Ned. Akad. v. Wetensch. 74 (1971), 135-145.

[Ta] A. D. Taĭmanov, 'On the extension of continuous mappings of topological spaces', Mat. Sb. 31 (1952), 459-462 (Russian).

Instituto de Matemáticas

Ciudad Universitaria (UNAM)

04510, México D.F.

México

e-mail: sgarcia@zeus.ccu.umich.mx
Escuela de Caminos Departamento de Matemática Aplicada Universidad Politécnica de Valencia 46071 Valencia Spain e-mail: sromague@mat.upv.es

Departament de Matemàtiques

Universitat Jaume I

Campus del Riu Sec

12071 Castelló

Spain

e-mail: sanchis@mat.uji.es 